\title{
Associations between hepatocyte growth factor, c-Met, and basic fibroblast growth factor and survival in endometrial cancer patients
}

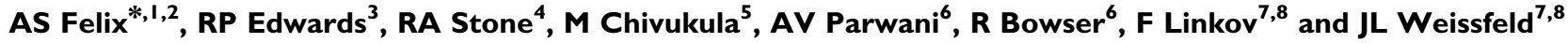

'Hormonal and Reproductive Epidemiology Branch, Division of Cancer Epidemiology and Genetics, National Cancer Institute, 6120 Executive Boulevard, Suite 550, Room 5006, Rockville, MD 20852-7234, USA; ${ }^{2}$ Cancer Prevention Fellowship Program, Center for Cancer Training, National Cancer Institute, Rockville, MD 20852, USA; ${ }^{3}$ Department of Obstetrics and Gynecologic Oncology, Magee-Womens Hospital of UPMC Health System, 300 Halket Street, Pittsburgh, PA 15213, USA; ${ }^{4}$ Department of Biostatistics, Graduate School of Public Health, University of Pittsburgh, Pittsburgh, PA I5261, USA; ${ }^{5}$ Department of Pathology, Magee-Womens Hospital of UPMC Health System, 300 Halket Street, Pittsburgh, PA 15213, USA; ${ }^{6}$ Department of Pathology, University of Pittsburgh Medical Center, Pittsburgh, PA 1526I, USA; ${ }^{7}$ Division of Cancer Prevention and Population Science, University of Pittsburgh Cancer Institute, 5150 Centre Avenue, Pittsburgh, PA 15232, USA; ${ }^{8}$ Department of Epidemiology, Graduate School of Public Health, University of Pittsburgh, Pittsburgh, PA 15261, USA
\end{abstract}

BACKGROUND: Hepatocyte growth factor (HGF), c-Met, and basic fibroblast growth factor (bFGF) are molecular markers that contribute to angiogenesis and proliferation in numerous cancers. We assessed the prognostic significance of these factors in tumour and stroma of endometrial cancer (EC) patients $(n=2 \mathrm{I} \mid)$.

METHODS: Immunohistochemistry (IHC) was used to detect tumour and stromal protein expression of the biomarkers. Associations between expression and clinicopathological factors were assessed using Chi-square tests. Kaplan-Meier curves, log-rank tests, and Cox regression were used to summarise associations between biomarker expression and overall survival (OS) and recurrence-free survival (RFS).

RESULTS: Tumour bFGF was significantly associated with high-grade endometrioid and clear cell histology $(P<0.00 \mathrm{I})$, advanced stage $(P=0.008)$, positive lymph-node involvement $(P=0.002)$, poor OS (log-rank test, $P=0.009)$, and poor RFS $(P<0.00 \mathrm{I})$. In multivariable analyses, cases with HGF-positive, stromal bFGF-positive tumours had a lower risk of death compared with cases with HGF-positive, stromal bFGF-negative tumours (hazard ratio (HR): 0.14, 95\% Cl: 0.03, 0.60). Cases with HGF-positive, bFGF-positive tumours had a higher risk of recurrence compared with cases with negative expression of both markers ( $\mathrm{HR}: 9.88,95 \% \mathrm{Cl}: 2.63$, 37.16).

CONCLUSION: These IHC data show that tumour and stromal bFGF expression have opposite associations with survival outcomes in EC patients. If confirmed in larger studies, tumour-derived bFGF could be an attractive target in EC therapy.

British Journal of Cancer (2012) I 06, 2004-2009. doi:I0.1038/bjc.20I2.200 www.bjcancer.com

Published online 22 May 2012

(C) 2012 Cancer Research UK

Keywords: prognostic biomarkers; angiogenesis; growth factors; recurrence-free survival; stroma

Angiogenesis, the formation of new vessels from preexisting parent vessels, is a critical factor in growth and dissemination of primary tumours (Folkman, 2002). During the multi-step development of cancer, tumour cells exploit the pathways controlled by angiogenic growth factors and their receptors in order to sustain expanding growth (Hanahan and Weinberg, 2011). Hepatocyte growth factor (HGF), also known as scatter factor, has mitogenic and motogenic effects on target epithelial and endothelial cells via its receptor c-Met (Yoshida et al, 2002). Overproduction of HGF by tumour cells or tumour-associated stromal cells and increased expression of the c-Met protein are two mechanisms that contribute to aberrant stimulation of this pathway. Consequences of dysregulated HGF and c-Met expression include tumour cell migration, proliferation, and protection from apoptosis (Comoglio et al, 2008). Overexpression of HGF and c-Met is common in numerous

*Correspondence: Dr AS Felix; E-mail: ashley.felix@gmail.com Received 17 February 2012; revised 12 April 2012; accepted 17 April 2012; published online 22 May 2012 cancer types and is associated with worse prognosis (Birchmeier et al, 2003). In addition, other pro-angiogenic factors secreted from tumour cells have been shown to induce HGF production through autocrine and paracrine mechanisms (Rosen et al, 1994; Nakamura et al, 1997). In several human cancer cell lines, basic fibroblast growth factor (bFGF) has been shown to be a potent stimulator of HGF production from stromal fibroblasts, promoting a cycle of continued growth and motility of tumour cells through HGF/c-Met activation (Rosen et al, 1994; Nakamura et al, 1997; Yoshida et al, 2002).

High-grade endometrial cancer (EC) subtypes, including highgrade endometrioid (EM), clear cell (CC), and papillary serous (PS), account for a disproportionate number of EC-related deaths each year (Hamilton et al, 2006). The role of angiogenic biomarkers in EC prognosis generally has been limited to EM tumours, although one previous study examined co-expression of HGF and c-Met in 38 PS cases; overall survival (OS) was significantly worse among the patients with strong HGF or strong c-Met expression, although no association between staining, myometrial invasion, or lymph-node involvement was observed 
(Bishop et al, 2011). In a case-series of 93 EM EC patients, Wagatsuma et al (1998) reported that c-Met expression was significantly associated with advanced stage, poor differentiation, and worse OS, whereas HGF was significantly associated with advanced stage. Neither c-Met nor HGF expression was an independent prognostic factor for survival in these EM cases (Wagatsuma et al, 1998). Two studies of associations between bFGF expression and tumour characteristics in EM EC patients have shown that strong expression of tumour-derived bFGF is significantly associated with high-grade tumours, presence of tumour necrosis, vascular invasion, and advanced stage tumours (Fujimoto et al, 1995; Stefansson et al, 2006). Furthermore, bFGF has been shown to significantly increase HGF transcription in EC cell lines (Yoshida et al, 2002).

This evidence suggests that bFGF expression is associated with aggressive endometrial tumour characteristics and is a potent inducer of HGF/c-Met interactions in EC cell lines. Whether or not bFGF is prognostically relevant for EC patients requires further investigation. Here, we examine the co-expression of bFGF, HGF, and c-Met in a histologically diverse sample of EC cases using immunohistochemistry (IHC). As divergent prognostic roles have been attributed to tumour and stromal expression of angiogenic biomarkers in other cancer sites, we evaluated these compartments separately (Donnem et al, 2007; Andersen et al, 2009). Our primary hypothesis is that tumour expression of the markers would be associated with worse survival, whereas stromal expression would be associated with better survival.

\section{MATERIALS AND METHODS}

\section{Study sample}

EC cases included in this study were treated at Magee-Womens Hospital in Pittsburgh, PA, between 1996 and 2008. Of 1486 eligible cases, we randomly sampled 211 cases using a stratified random sampling design. The cohort of eligible cases has been described previously (Felix et al, 2010). Histology subtype (EM (any grade) and non-EM (CC + PS)) and International Federation of Gynecology and Obstetrics (FIGOs) stage (stage I, stage II, stage III, and stage IV) defined the eight sampling strata within which simple random samples were drawn. Among the EM cases, our sample included 33 stage I, 29 stage II, 37 stage III, and 15 stage IV cases. Among non-EM cases, our sample included 40 stage I, 9 stage II, 26 stage III, and 22 stage IV cases. Our goal was to include 25 cases per stratum, however, because of insufficient tumour blocks in certain strata, larger strata were oversampled. This study was approved by the University of Pittsburgh Institutional Review Board. At the time of surgery, all the patients provided written informed consent for use of tissue samples in future research studies.

\section{IHC}

Formalin-fixed, paraffin-embedded archival tissue samples were retrieved from the 211 sampled cases. Tissue material was collected from the primary tumour; metastatic tissues were not available for this study. Prior to slide sectioning, tissue blocks and matching hematoxylin- and eosin-stained slides were reviewed by one expert gynaecologic pathologist to select tumour regions with ample tumour and stromal material. The slides were deparaffinised and hydrated with xylenes and washed with progressively decreasing alcohol concentrations. Endogenous peroxidase activity was blocked with $3 \%$ methanol peroxide. After antigen retrieval in $0.01 \mathrm{~m}$ boiling citrate buffer ( $\mathrm{pH}$ 6.0) in a microwave, non-specific staining was blocked with protein block (Dako North America, Inc, Carpinteria, CA, USA). Whole-tissue sections were incubated for $1 \mathrm{~h}$ at room temperature with $100 \mu \mathrm{l}$ of polyclonal HGF antibody
(R\&D Systems, Minneapolis, MN, USA, dilution $1: 30$ ), polyclonal c-Met antibody (Santa Cruz Biotechnology Inc, Santa Cruz, CA, USA, dilution 1:75), and polyclonal bFGF antibody (Millipore, Billerica, MA, USA, dilution $1: 1000$ ). Slides were subsequently rinsed in PBS/Tween solution for $5 \mathrm{~min}$ followed by incubation with a polymer (ImmPRESS Universal reagent; Vector Laboratories, Burlingame, CA, USA) followed by development with diaminobenzidine for detection. Slides were counterstained with Shandon Hematoxylin for $2 \mathrm{~min}$, rinsed in several concentrations of alcohol solution, mounted, and viewed. Positive and negative controls were run with each batch of approximately 40 slides. Placenta, fibroadenoma, and granulation tissue were the positive controls for HGF, c-Met, and bFGF, respectively. Omission of the primary antibody was used to control for any immunoreactivity due to secondary antibody alone. Cytoplasmic and nuclear immunostaining was observed for HGF and bFGF; cytoplasmic staining was observed for c-Met. To validate our IHC findings, we randomly selected a subset of 20 cases for re-analysis. New slides were cut from tumour blocks and stained with antibodies. This subset of slides was evaluated without knowledge of the previously recorded scores and the intra-rater agreement was $100 \%$.

IHC expression of tumour and stromal HGF, c-Met, and bFGF was initially evaluated using a semi-quantitative score. The intensity of staining $(0=$ none, $1=$ weak, $2=$ moderate, and $3=$ strong) and the proportion of positive-staining cells $(1=1-5 \%$, $2=6-20 \%, 3=21-80 \%$, and $4=>80 \%$ ) were summed to calculate the cumulative score. Acceptable values of the cumulative score are $0,2,3,4,5,6$, and 7 . As the prevalence of positive expression scores (cumulative scores 2-7) was low for HGF and c-Met, we chose to categorise expression as negative (0) vs positive (2-7). An expert gynaecologic pathologist blinded to survival outcomes evaluated all the slides. Furthermore, tumour and stromal localisation was analysed from a single slide. Stromal expression of HGF and c-Met was negative in all cases, whereas bFGF expression was apparent in the tumour and stroma.

\section{Statistical analyses}

Associations between HGF, c-Met, bFGF, and clinicopathological variables and associations between pair of biomarkers were assessed using Chi-square tests. OS and recurrence-free survival (RFS) were quantified as the number of days between the date of diagnosis and the date of death from all the causes or date of recurrence, respectively. Patients who did not experience either outcome were censored at the last date of contact or the date of last follow-up (December 31, 2008). RFS analyses included only cases that were known to be disease-free following the primary surgery. Kaplan-Meier estimates of OS and RFS and log-rank tests were used to compare survival distributions by biomarker expression status.

Associations between HGF, c-Met, and bFGF and survival outcomes were adjusted for histology subtype, FIGO stage, and age using Cox proportional hazards models. In addition to main effect models, we examined pairwise interactions between the biomarkers. All the Cox model analyses were stratified by year of diagnosis. Sampling weights were calculated as the inverse probability of being selected into this study from the larger sampling frame, given the availability of tumour blocks. In Stata 11 , the svy family of commands was used to incorporate sampling weights (StataCorp LP, College Station, TX, USA).

\section{RESULTS}

Examples of the IHC expression of HGF, c-Met, and bFGF (tumour and stromal) are shown in Figure 1. Positive expression of HGF, c-Met, tumour bFGF, and stromal bFGF was observed in $31(15 \%)$, $56(26 \%), 35(16 \%)$, and $116(55 \%)$ cases, respectively. 

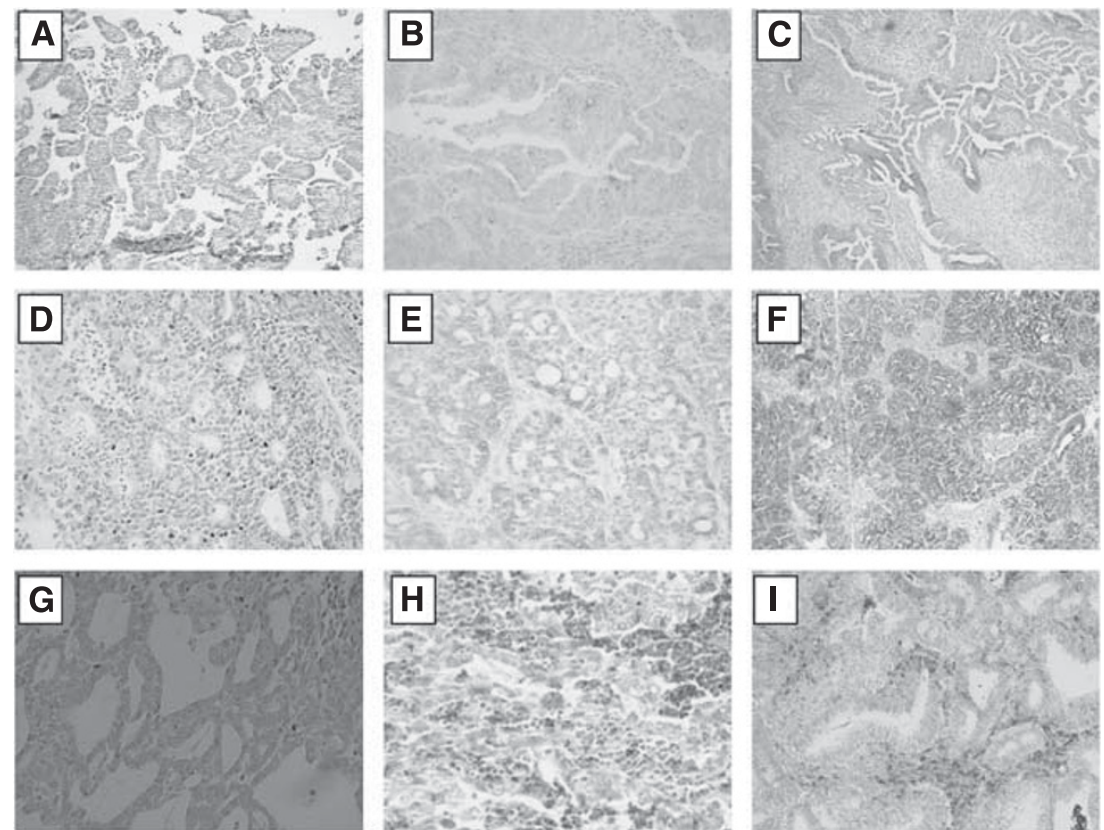

Figure I Representative IHC stains showing $(\mathbf{A})$ negative HGF expression, (B and $\mathbf{C})$ positive HGF expression, $(\mathbf{D})$ negative c-Met expression, $(\mathbf{E}$ and $\mathbf{F})$ positive c-Met, $(\mathbf{G})$ negative bFGF expression, $(\mathbf{H})$ positive tumour bFGF expression, and (I) positive stromal bFGF expression.

No significant associations were observed between HGF or c-Met expression and any of the clinicopathological factors shown in Table 1 ( $P>0.12$ for each). Tumour bFGF was significantly associated with histology type, FIGO stage, lymph-node involvement, and metastasis. Compared with low-grade EM cases (1\%), positive tumour bFGF expression was significantly more common in high-grade EM (32\%), CC (32\%), and PS cases (16\%). Advanced FIGO stage cases had a significantly higher prevalence of positive tumour bFGF expression (18\%) than early-stage cases (5\%). Additionally, tumour bFGF expression was higher in cases with positive lymph-node involvement $(33 \%)$ compared with cases with negative lymph-node involvement $(8 \%)$ or no nodal examination $(2 \%)$, and significantly more common among metastatic cases (24\%) than among non-metastatic cases (6\%). Stromal bFGF was borderline significantly associated with histology subtype $(P=0.06)$. Stromal bFGF expression was more common among CC cases (70\%), high-grade EM (61\%), and low-grade EM (59\%) compared with PS cases (44\%). When examining the association between pairs of biomarkers, only HGF and c-Met were significantly associated with each other. c-Met positivity was significantly higher among cases with positive HGF expression (63\%) compared with cases with negative HGF expression (15\%, $P=0.03$, data not tabled).

Median follow-up time was 1287 days in the sample (range: 24-4659 days) and 1413 days in the sampling frame (range: 1-4887 days). Overall, patients with positive tumour bFGF expression had significantly worse OS $(P=0.009)$ and RFS $(P<0.001)$ compared with patients with negative tumour bFGF expression (Table 2; Figures $2 \mathrm{~A}$ and B). HGF, c-Met, and stromal bFGF expression were not significantly associated with OS or RFS.

In the multivariable Cox proportional hazards models, we observed significant interactions between HGF and stromal bFGF expression for OS $(P=0.02$, degrees of freedom $=1)$ and between HGF and tumour bFGF for RFS $(P=0.007$, degrees of freedom $=1$; Table 3, Figure 3). Relative to cases who were negative on both biomarkers, the hazard of death was nonsignificantly elevated for HGF-positive, stromal bFGF-negative cases $(\mathrm{HR}=2.09)$ and nonsignificantly reduced for HGF-positive, stromal bFGF-positive cases $(\mathrm{HR}=0.29)$. However, HGF-positive, stromal bFGF-positive cases had a significantly reduced risk of death compared with
HGF-positive, stromal bFGF-negative cases (HR: 0.29/2.09 $=0.14$, $95 \%$ CI 0.03, 0.60). Among the 18 cases with HGF-positive, stromal bFGF-positive expression, 10 had low-grade EM tumours, 9 had early-stage tumours, and 15 had no evidence of metastases (data not tabled).

In the RFS model (Table 3), based on very small numbers HGFpositive, tumour bFGF-positive patients had an almost 10 times higher risk of recurrence compared with patients with negative expression of both biomarkers (HR: 9.88, 95\% CI 2.63, 37.16), whereas HGF-positive, tumour bFGF-negative patients had a significantly lower risk of recurrence (HR: $0.07,95 \%$ CI 0.00 , 0.81 ). Among the five cases with an HGF-positive, tumour bFGFpositive expression pattern, four had either a CC or PS tumour, two had late-stage tumours, and two had evidence of metastases (data not tabled).

\section{DISCUSSION}

We examined the expression of HGF, c-Met, and bFGF using IHC in a large sample of EC cases and correlated expression with clinicopathological factors and patient outcomes. OS was significantly associated with stromal bFGF expression in the presence of HGF expression. Specifically, HGF-positive, stromal bFGFpositive patients had significantly better OS compared with HGFpositive, stromal bFGF-negative patients. The small group of patients who were HGF-positive, tumour bFGF-positive had almost a 10 times higher risk of recurrence compared with tumour HGFnegative, tumour bFGF-negative patients. Additionally, patients who were HGF-positive and tumour bFGF-negative had a significantly reduced risk of recurrence compared with HGFnegative, tumour bFGF-negative patients. To our knowledge, this is the first study to indicate prognostic significance of these angiogenic factors in EC patients.

Interactions between tumour cells and tumour-associated stromal cells are hypothesised to have a role in aggressive cancer phenotypes. The host stromal response is activated initially to eliminate tumour cells; however, tumour cells co-opt the functions of recruited stromal cells to enhance the growth and invasion of the tumour, resulting in a tumour-supportive stroma (De Wever 
Table I Associations between HGF, c-Met, and bFGF expression and clinicopathological factors

Positive expression, $n^{\mathrm{a}}(\%)^{\mathrm{b}}$

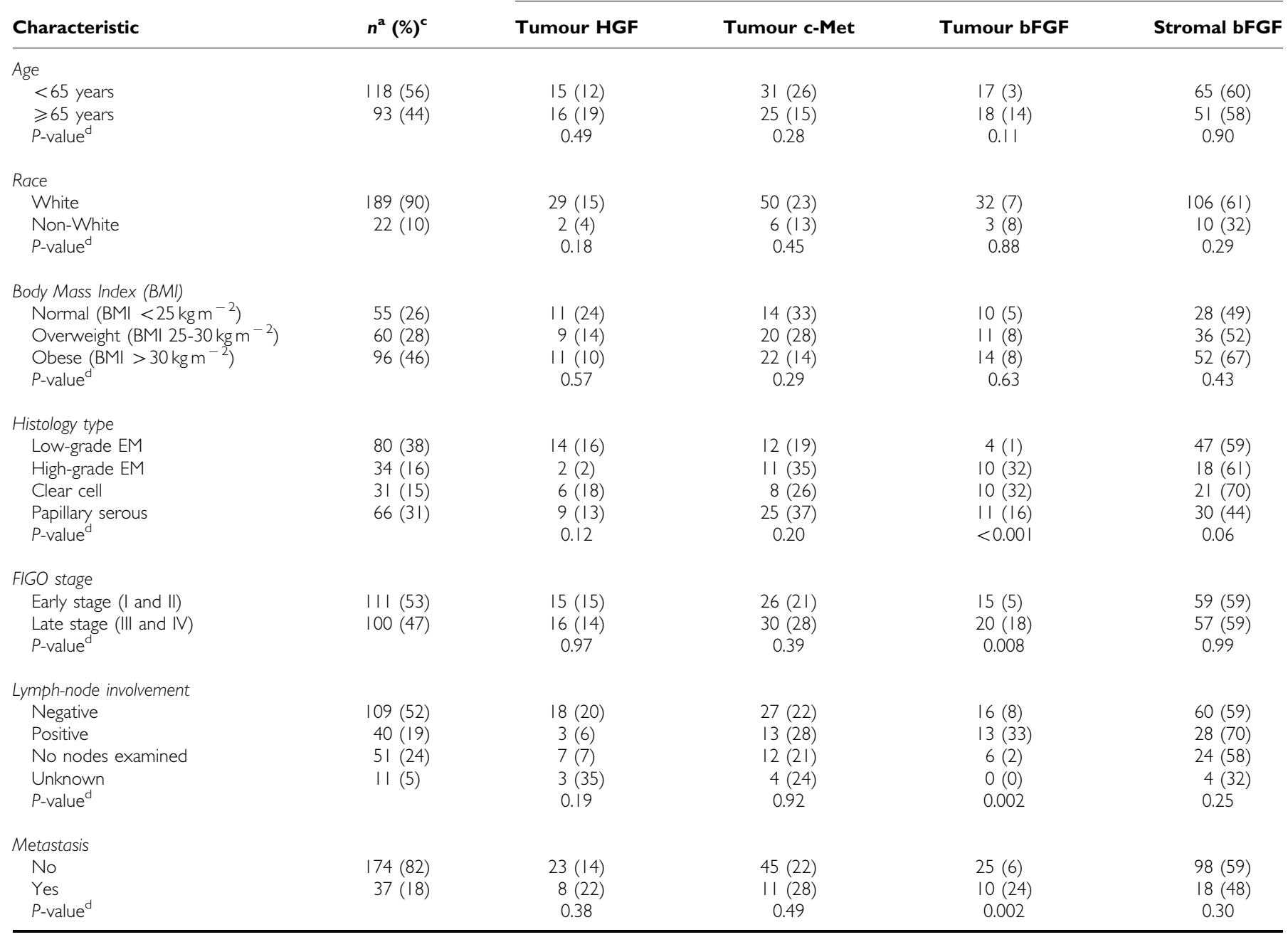

a'Sample count. 'Weighted proportion in the sampling frame, $n=1486$. 'Unweighted proportion in the study sample, $n=211$. 'Adjusted Wald's $P$-value.

Table 2 Summary of mortality and recurrence by HGF, c-Met, tumour bFGF, and stromal bFGF status

\begin{tabular}{|c|c|c|c|c|}
\hline $\begin{array}{l}\text { Biomarker } \\
\text { expression }\end{array}$ & $\begin{array}{l}\text { Deaths/n } \\
(87 / 211)\end{array}$ & $P$-value & $\begin{array}{c}\text { Recurrences/ } \\
n(42 / 174)\end{array}$ & $P$-value ${ }^{*}$ \\
\hline $\begin{array}{l}\text { HGF } \\
\text { Negative } \\
\text { Positive }\end{array}$ & $\begin{array}{l}76 / 180 \\
\mid 1 / 31\end{array}$ & 0.96 & $\begin{array}{c}38 / 150 \\
4 / 24\end{array}$ & 0.21 \\
\hline $\begin{array}{l}\text { c-Met } \\
\text { Negative } \\
\text { Positive }\end{array}$ & $\begin{array}{l}61 / 155 \\
26 / 56\end{array}$ & 0.48 & $\begin{array}{l}28 / 130 \\
14 / 44\end{array}$ & 0.14 \\
\hline $\begin{array}{c}\text { Tumour bFGF } \\
\text { Negative } \\
\text { Positive }\end{array}$ & $\begin{array}{l}67 / 176 \\
20 / 35\end{array}$ & 0.009 & $\begin{array}{l}30 / 147 \\
12 / 27\end{array}$ & $<0.001$ \\
\hline $\begin{array}{c}\text { Stromal bFGF } \\
\text { Negative } \\
\text { Positive }\end{array}$ & $\begin{array}{c}43 / 95 \\
44 / 116\end{array}$ & 0.83 & $\begin{array}{l}19 / 75 \\
23 / 99\end{array}$ & 0.22 \\
\hline
\end{tabular}

*P-values based on log-rank statistics.

and Mareel, 2003; Joyce and Pollard, 2009). Furthermore, as the metabolic demand for nutrients increases, tumour cells activate angiogenesis through secretion of various cytokines. Therefore, understanding the role of the tumour- and stromal-derived
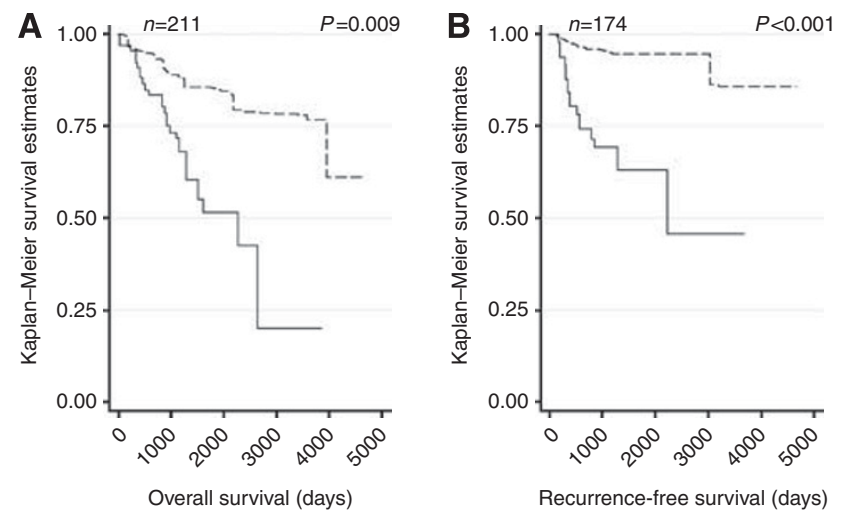

Figure 2 Kaplan-Meier curves of (A) OS and (B) RFS by tumour bFGF status.

angiogenic pathways is important for targeted therapy strategies (Joyce, 2005; Albini and Sporn, 2007; Chung et al, 2010).

The underlying mechanism describing a protective role of stromal-derived bFGF is unknown. This study, along with others, has noted that angiogenic proteins expressed in the stroma have a 
Table 3 Cox proportional hazards model for overall survival and recurrence-free survival showing interactions between HGF and stromal bFGF and HGF and tumour bFGF, respectively

\begin{tabular}{|c|c|c|c|}
\hline Overall Survival $(n=2 I I)$ & Deaths/n & $\operatorname{HR}(95 \% \mathrm{CI})^{\mathrm{a}}$ & $P^{\mathbf{b}}$ \\
\hline HGF-negative, stromal bFGF-negative & $36 / 82$ & I.00 (Reference) & \\
\hline HGF-positive, stromal bFGF-positive & $4 / 18$ & $0.29(0.06,1.33)$ & 0.11 \\
\hline Recurrence-free survival $(n=174)$ & Recurrences/n & $\operatorname{HR}(95 \% \mathrm{Cl})^{\mathrm{a}}$ & $P^{\mathbf{b}}$ \\
\hline HGF-negative, tumour bFGF-negative & $29 / 128$ & 1.00 (Reference) & \\
\hline HGF-positive, tumour bFGF-negative & $1 / 19$ & $0.07(0.00,0.81)$ & 0.03 \\
\hline HGF-negative, tumour bFGF-positive & $9 / 22$ & $1.56(0.44,5.53)$ & 0.49 \\
\hline HGF-positive, tumour bFGF-positive & $3 / 5$ & $9.88(2.63,37.16)$ & 0.001 \\
\hline
\end{tabular}

${ }^{a}$ Adjusted for c-Met, stage, histology subtype, and age; stratified by year of diagnosis. ${ }^{b}$ Adjusted Wald's $P$-value. ${ }^{c}$ Three degree of freedom $P$-value for heterogeneity
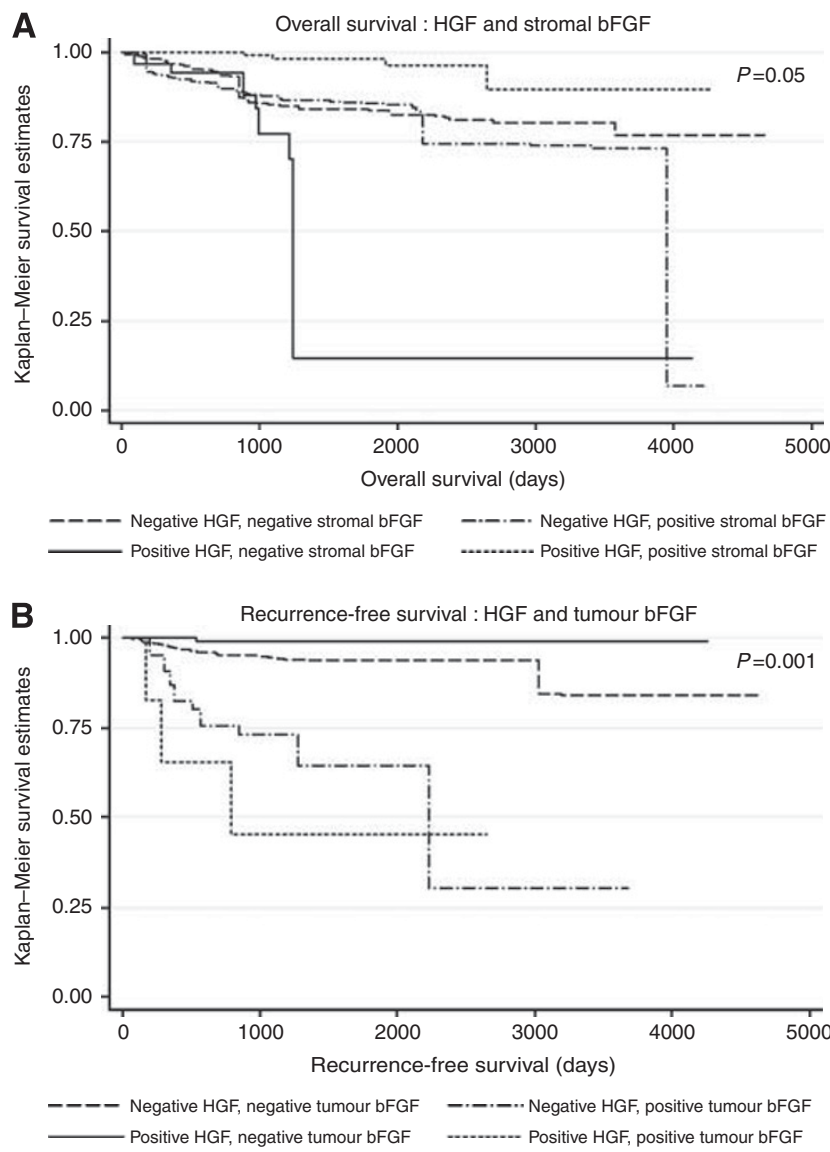

Figure 3 Kaplan-Meier curves of (A) OS by HGF and stromal bFGF and (B) RFS by HGF and tumour bFGF.

divergent prognostic role compared with tumour expression of the same proteins. In two independent non-small cell lung cancer (NSCLC) cohorts, stromal bFGF expression was inversely associated with lymph-node metastasis, advanced stage, and diseasespecific mortality, indicating a potentially protective role of this cytokine (Guddo et al, 1999; Andersen et al, 2009). Conversely, Takanami et al (1996) reported that tumour bFGF expression was significantly associated with poor prognosis in NSCLC patients. In another NSCLC cohort, Donnem et al (2007) reported that stromal expression of vascular endothelial growth-factor ligands and receptors was associated with better survival in NSCLC patients, whereas tumour expression of the same biomarkers was associated with worse prognosis.

Our findings agree with the body of literature reporting an association between tumour bFGF expression, aggressive clinicopathological characteristics, and poor prognosis in various cancers (Yamazaki et al, 1997; Faridi et al, 2002; Strohmeyer et al, 2004; Behrens et al, 2008; Alshenawy, 2010). In this study, tumour bFGF was significantly associated with aggressive endometrial histology subtypes, advanced stage, positive lymph-node involvement, and worse RFS. We propose that a delicate balance between tumourinhibiting and tumour-promoting effects of bFGF may exist: although the host stroma controls expression of bFGF, anti-cancer effects may be dominant; however, when the tumour becomes independent of stromal paracrine factors through the establishment of autocrine bFGF stimulation, poor outcomes are more likely to occur.

We also observed a statistically significant association between HGF and c-Met expression. The biological effects of HGF are mediated by the c-Met receptor, which is frequently overexpressed in many human cancers (Birchmeier et al, 2003). Upon HGF binding, c-Met activates a number of cellular responses, including motility or scattering of epithelial cells, proliferation, and invasion (Birchmeier et al, 2003). Despite the importance of HGF in c-Met activation, most prognostic studies focus solely on the aberrant expression of c-Met. In fact, development and implementation of HGF antagonists lag far behind the use of c-Met inhibitors currently used in clinical trials (Yap and de Bono, 2010). Evaluation of the co-expression of HGF and c-Met may lead to a better understanding of the overall importance of this signalling pathway in cancer prognosis.

Strengths of this study include high quality pathology data, discrimination between tumour and stromal expression of the biomarkers, and an evaluation of the prognostic role of this biomarker pathway using multivariable methods, with appropriate weighting for tumour-block availability within the sampling frame. Despite including 211 EC patients, the major limitation of this study is the sample size, particularly the small number of HGFpositive patients in the RFS analysis. Furthermore, the prevalence of positive HGF and c-Met expression in our study is lower than previously published EC studies. We observed positive HGF and c-Met expression in $15 \%$ and $22 \%$ of EC cases compared with $90 \%$ and $63 \%$ of the tumours in the Wagatsuma series and $100 \%$ and $87 \%$ of PS EC cases in the Bishop series, respectively (Wagatsuma et al, 1998; Bishop et al, 2011). The low positive prevalence of biomarkers in our study may reflect variations in IHC procedures across laboratories, subjective assessment of the IHC slides, or differences in case selection. We note that our study sample was selected based on tumour characteristics (stage and histology 
subtype), which differs from the use of consecutively treated EM EC cases as in the Wagatsuma series or serous EC cases included in the Bishop series. Additionally, our use of whole-section slides rather than tissue microarrays may have contributed to differences between our study and Bishop et al.

In addition to replicating the results of this study, future examinations of bFGF should examine the mechanisms that could explain the apparently protective role of stromal expression. Functional studies that can provide a biological rationale for our observed associations would be useful in recommending molecularly targeted therapies. Recent evidence supports a role for therapeutic targeting of the normal cells that surround the primary tumour, that is, the tumour microenvironment (Joyce, 2005; Hiscox et al, 2011). Compared with neoplastic cells, which are characterised by genetic mutations, cells of the microenvironment are genetically stable and may provide a more attractive therapeutic target. Several novel agents targeting HGF and bFGF, including AMG102 and AZD4547, respectively, are currently in phase I and II clinical trials (Gordon et al, 2010; Lieu et al, 2011).

In summary, this study suggests the presence of a significant interaction between HGF and bFGF expression in EC survival. The different association between survival and tumour $v s$ stromal expression of bFGF is indicative of this marker's diverse role in tumour physiology and has potential implications for targeted therapies.

\section{ACKNOWLEDGEMENTS}

This research was supported by a National Institutes of Health grant R25-CA057703.

\section{REFERENCES}

Albini A, Sporn MB (2007) The tumour microenvironment as a target for chemoprevention. Nat Rev Cancer 7(2): 139-147

Alshenawy HA (2010) Prognostic significance of vascular endothelial growth factor, basic fibroblastic growth factor, and microvessel density and their relation to cell proliferation in B-cell non-Hodgkin's lymphoma. Ann Diagn Pathol 14(5): 321-327

Andersen S, Donnem T, Al-Saad S, Al-Shibli K, Busund LT, Bremnes RM (2009) Angiogenic markers show high prognostic impact on survival in marginally operable non-small cell lung cancer patients treated with adjuvant radiotherapy. J Thorac Oncol 4(4): 463-471

Behrens C, Lin HY, Lee JJ, Raso MG, Hong WK, Wistuba II, Lotan R (2008) Immunohistochemical expression of basic fibroblast growth factor and fibroblast growth factor receptors 1 and 2 in the pathogenesis of lung cancer. Clin Cancer Res 14(19): 6014-6022

Birchmeier C, Birchmeier W, Gherardi E, Vande Woude GF (2003) Met, metastasis, motility and more. Nat Rev Mol Cell Biol 4(12): 915-925

Bishop EA, Lengyel ER, Yamada SD, Montag A, Temkin SM (2011) The expression of hepatocyte growth factor (HGF) and c-Met in uterine serous carcinoma. Gynecol Oncol 121(1): 218-223

Chung AS, Lee J, Ferrara N (2010) Targeting the tumour vasculature: insights from physiological angiogenesis. Nat Rev Cancer 10(7): 505-514

Comoglio PM, Giordano S, Trusolino L (2008) Drug development of MET inhibitors: targeting oncogene addiction and expedience. Nat Rev Drug Discov 7(6): 504-516

De Wever O, Mareel M (2003) Role of tissue stroma in cancer cell invasion. J Pathol 200(4): 429-447

Donnem T, Al-Saad S, Al-Shibli K, Delghandi MP, Persson M, Nilsen MN, Busund LT, Bremnes RM (2007) Inverse prognostic impact of angiogenic marker expression in tumor cells $v s$ stromal cells in non small cell lung cancer. Clin Cancer Res 13(22 Part 1): 6649-6657

Faridi A, Rudlowski C, Biesterfeld S, Schuh S, Rath W, Schroder W (2002) Long-term follow-up and prognostic significance of angiogenic basic fibroblast growth factor (bFGF) expression in patients with breast cancer. Pathol Res Pract 198(1): 1-5

Felix AS, Weissfeld JL, Stone RA, Bowser R, Chivukula M, Edwards RP, Linkov F (2010) Factors associated with type I and type II endometrial cancer. Cancer Causes Control 21(11): 1851-1856

Folkman J (2002) Role of angiogenesis in tumor growth and metastasis. Semin Oncol 29(6 Suppl 16): 15-18

Fujimoto J, Hori M, Ichigo S, Tamaya T (1995) Expression of basic fibroblast growth factor and its mRNA in uterine endometrial cancers. Invasion Metastasis 15(5-6): 203-210

Gordon MS, Sweeney CS, Mendelson DS, Eckhardt SG, Anderson A, Beaupre DM, Branstetter D, Burgess TL, Coxon A, Deng H, Kaplan-Lefko P, Leitch IM, Oliner KS, Yan L, Zhu M, Gore L (2010) Safety, pharmacokinetics, and pharmacodynamics of AMG 102, a fully human hepatocyte growth factor-neutralizing monoclonal antibody, in a first-inhuman study of patients with advanced solid tumors. Clin Cancer Res 16(2): 699-710

Guddo F, Fontanini G, Reina C, Vignola AM, Angeletti A, Bonsignore G (1999) The expression of basic fibroblast growth factor (bFGF) in tumorassociated stromal cells and vessels is inversely correlated with non-small cell lung cancer progression. Hum Pathol 30(7): 788-794

Hamilton CA, Cheung MK, Osann K, Chen L, Teng NN, Longacre TA, Powell MA, Hendrickson MR, Kapp DS, Chan JK (2006) Uterine papillary serous and clear cell carcinomas predict for poorer survival compared to grade 3 endometrioid corpus cancers. Br J Cancer 94(5): 642-646

Hanahan D, Weinberg RA (2011) Hallmarks of cancer: the next generation. Cell 144(5): 646-674

Hiscox S, Barrett-Lee P, Nicholson RI (2011) Therapeutic targeting of tumor-stroma interactions. Expert Opin Ther Targets 15(5): 609-621

Joyce JA (2005) Therapeutic targeting of the tumor microenvironment. Cancer Cell 7(6): 513-520

Joyce JA, Pollard JW (2009) Microenvironmental regulation of metastasis. Nat Rev Cancer 9(4): 239-252

Lieu C, Heymach J, Overman M, Tran H, Kopetz S (2011) Beyond VEGF: inhibition of the fibroblast growth factor pathway and antiangiogenesis. Clin Cancer Res 17(19): 6130-6139

Nakamura T, Matsumoto K, Kiritoshi A, Tano Y (1997) Induction of hepatocyte growth factor in fibroblasts by tumor-derived factors affects invasive growth of tumor cells: in vitro analysis of tumor-stromal interactions. Cancer Res 57(15): 3305-3313

Rosen EM, Joseph A, Jin L, Rockwell S, Elias JA, Knesel J, Wines J, McClellan J, Kluger MJ, Goldberg ID, Zitnik R (1994) Regulation of scatter factor production via a soluble inducing factor. J Cell Biol 127(1): 225-234

Stefansson IM, Salvesen HB, Akslen LA (2006) Vascular proliferation is important for clinical progress of endometrial cancer. Cancer Res 66(6): 3303-3309

Strohmeyer D, Strauss F, Rossing C, Roberts C, Kaufmann O, Bartsch G, Effert P (2004) Expression of bFGF, VEGF and c-met and their correlation with microvessel density and progression in prostate carcinoma. Anticancer Res 24(3a): 1797-1804

Takanami I, Tanaka F, Hashizume T, Kikuchi K, Yamamoto Y, Yamamoto T, Kodaira S (1996) The basic fibroblast growth factor and its receptor in pulmonary adenocarcinomas: an investigation of their expression as prognostic markers. Eur J Cancer 32A(9): 1504-1509

Wagatsuma S, Konno R, Sato S, Yajima A (1998) Tumor angiogenesis, hepatocyte growth factor, and c-Met expression in endometrial carcinoma. Cancer 82(3): 520-530

Yamazaki K, Nagao T, Yamaguchi T, Saisho H, Kondo Y (1997) Expression of basic fibroblast growth factor (FGF-2)-associated with tumour proliferation in human pancreatic carcinoma. Virchows Arch 431(2): 95-101

Yap TA, de Bono JS (2010) Targeting the HGF/c-Met Axis: State of Play. Mol Cancer Ther 9(5): 1077-1079

Yoshida S, Harada T, Iwabe T, Taniguchi F, Fujii A, Sakamoto Y Yamauchi N, Shiota G, Terakawa N (2002) Induction of hepatocyte growth factor in stromal cells by tumor-derived basic fibroblast growth factor enhances growth and invasion of endometrial cancer. J Clin Endocrinol Metab 87(5): 2376-2383

This work is published under the standard license to publish agreement. After 12 months the work will become freely available and the license terms will switch to a Creative Commons Attribution-NonCommercial-Share Alike 3.0 Unported License. 